\title{
On Optimal Input Signal Design for Frequency Response Estimation
}

\author{
Bo Wahlberg, Håkan Hjalmarsson and Petre Stoica
}

\begin{abstract}
This paper studies optimal input excitation design for parametric frequency response estimation. The objective is to minimize the uncertainty of functions of the frequency response estimate at a specified frequency $\omega$ while limiting the power of the input signal. We focus on least-squares estimation of Finite Impulse Response (FIR) models and minimum variance input design. The optimal input problem is formulated as a convex optimization problem (semi-definite program) in the second order statistics of the input signal. We analytically characterize the optimal solution for first order FIR systems with two parameters, and perform a numerical study to obtain insights in the optimal solution for higher order models. The optimal solution is compared to the case when a sinusoidal input signal, with frequency $\omega$ and amplitude that gives the same accuracy as the optimal input, is used as excitation signal. For first order FIR models with two parameters the input signal power can be reduced at best by a factor of two by using the optimal input signal compared with such a sinusoidal input signal. Numerical studies show that less is in general gained for higher order systems, for which a sinusoidal input signal with frequency $\omega$ often is optimal. We consider estimation of the $\mathscr{H}_{\infty}$-norm of a stable linear system, that is the maximum of the absolute value of the corresponding frequency response. An asymptotic error variance expression for $\mathscr{H}_{\infty}$-norm estimates is derived.
\end{abstract}

\section{INTRODUCTION}

Consider a scalar discrete time asymptotically stable timeinvariant linear dynamical system with impulse response sequence $\left\{g_{k}\right\}$, input signal sequence $\{u(t)\}$ and output signal sequence $\{y(t)\}$, and additive zero mean white noise $\{e(t)\}$ with variance $\lambda_{e}$. We then have

$$
y(t)=\sum_{k=0}^{\infty} g_{k} u(t-k)+e(t) .
$$

The corresponding frequency response function is defined by

$$
G\left(e^{i \omega}\right)=\sum_{k=0}^{\infty} g_{k} e^{-i \omega k}, \quad \omega \in(-\pi, \pi], \quad i=\sqrt{-1},
$$

and is a most important tool in feedback control design. The $\mathscr{H}_{\infty}$-norm of a stable system equals the maximum of the absolute value of $G\left(e^{i \omega}\right)$. A sinusoidal input signal with the corresponding peak frequency maximizes the input to output $\mathscr{L}_{2}$-gain of the system, see e.g. [4].

Frequency response functions estimation is a fundamental problem in both spectral estimation and system identification, see e.g. [14] and [6]. For linear time invariant single-input single-output systems, it is shown in [11] that a water-bed effect holds for the asymptotic variance of the frequency response estimate, $\operatorname{As} \operatorname{Var}\left\{\hat{G}\left(e^{i \omega}\right)\right\}$. For output error models,

This work was partially supported by the Swedish Research Council and the Linnaeus Center ACCESS at KTH

B. Wahlberg and H. Hjalmarsson are with the Automatic Control Lab and ACCESS, School of Electrical Engineering, KTH, SE-100 44 Stockholm, Sweden. (e-mail: bo.wahlberg@ee.kth.se, hakan.hjalmarsson@ee.kth.se.)

P. Stoica is with the Department of Information Technology, Uppsala University, SE-75 105 Uppsala, Sweden (e-mail: ps@it.uu.se). including FIR models, the result is

$$
\frac{1}{2 \pi} \int_{-\pi}^{\pi} \operatorname{As} \operatorname{Var}\left\{\hat{G}\left(e^{i \omega}\right)\right\} \Phi_{u}(\omega) d \omega=\frac{\lambda_{e} n_{u}}{N},
$$

where $\Phi_{u}(\omega)$ is the input power spectral density, $\lambda_{e}$ is the noise variance, $n_{u}$ is the number of identifiable parameters (typically $n+1$ for an $n$ :th order FIR model), and $N$ is the number of observations. This result should be compared to the classical frequency function variance expression, [8],

$$
\operatorname{As} \operatorname{Var}\left\{\hat{G}\left(e^{i \omega}\right)\right\} \approx \frac{\lambda_{e}(n+1)}{N \Phi_{u}(\omega)},
$$

which is only valid for for large model orders $n$. The paper [3] presents a finite sample variance expression for fixed model orders and periodic input signals that is very similar to (3). It also contains a more recent survey on variance expressions for estimated frequency functions.

If it is possible to choose the excitation in the data collection experiment, an interesting idea is to find the input signal that minimizes the uncertainty $\operatorname{AsVar}\left\{\hat{G}\left(e^{i \omega}\right)\right\}$. This makes sense only if we constrain, for example, the power of the input signal as otherwise the error can be made arbitrarily small. The problem of finding optimal input signals for parameter estimation in dynamic systems is a classical topic. An excellent survey of the field up to 1974 is given in [9]. There has recently been a renewed interest in the optimal input design problem, and a recent survey of the state of the art is given in [2]. We will use the least costly identification approach introduced in [1]. The paper [10] has recently showed the equivalence of least costly and traditional experiment design.

The outline of this paper is as follows. Section II contains a variance analysis of identified FIR models and corresponding frequency functions. Optimal input design problems for frequency function estimation are presented in Section III, which also contains explicit expression for the optimal solution for first order FIR systems. The general case is evaluated by a simulation study in Section IV, while Section $\mathrm{V}$ concludes the paper

\section{VARIANCE EXPRESSIONS}

We will consider identification of $n$ :th order finite impulse response (FIR) systems, for which $g_{k}=0, k>n$. Furthermore, it will be assumed that $u(t)=0$ for $t \leq 0$ and $t>N$, i.e. zero initial and final conditions. The input to output relation (1) for a given input sequence $\{u(t), t=1, \ldots, N\}$ can then be written in matrix notation as

$$
\begin{aligned}
& \mathbf{y}=\mathbf{U g}+\mathbf{e}, \\
& \mathbf{y}=[y(1), y(2), \ldots, y(N+n)]^{T}, \\
& \mathbf{g}=\left[g_{0}, g_{1}, \ldots, g_{n}\right]^{T}, \\
& \mathbf{e}=[e(1), e(2), \ldots, e(N+n)]^{T},
\end{aligned}
$$


and $\mathbf{U}$ is a $n+N$ times $n+1$ lower triangular Toeplitz matrix with first column $\mathbf{u}=[u(1), u(2), \ldots u(N), 0, \ldots, 0]^{T}$. If the zero initial or the zero final conditions are not satisfied, the first $n$ or the last $n$ rows of $\mathbf{U}$ and $\mathbf{y}$ should be removed for (4) to hold.

The least squares estimate of the impulse response vector $\mathrm{g}$ is

$$
\hat{\mathbf{g}}=\left[\frac{1}{N} \mathbf{U}^{T} \mathbf{U}\right]^{-1}\left[\frac{1}{N} \mathbf{U}^{T} \mathbf{y}\right] .
$$

Here we estimate $n+1$ parameters from at best $N+n$ observations. Typically, for $N$ observations the number of parameters, $n+1$, needs to be at most on the order of $\sqrt{N / \log N}$ for almost sure convergence, see [7].

The statistical properties of $\hat{\mathbf{g}}$ are well known, see e.g. [6]. We will use $\mathbf{g}_{o}$ to denote the true systems parameters, and $\Delta \mathbf{g}=\hat{\mathbf{g}}-\mathbf{g}_{o}$ for the corresponding estimation error. The covariance matrix of the least squares estimate error equals

$$
\operatorname{Cov}\{\hat{\mathbf{g}}\}=\mathrm{E}\left\{\Delta \mathbf{g} \Delta \mathbf{g}^{T}\right\}=\mathbf{P}_{N}, \quad \mathbf{P}_{N}=\frac{\lambda_{e}}{N}\left[\frac{1}{N} \mathbf{U}^{T} \mathbf{U}\right]^{-1} .
$$

Note that $\mathbf{P}_{N}$ corresponding to (4) is proportional to the inverse of a symmetric Toeplitz matrix with elements

$$
\left[\frac{1}{N} \mathbf{U}^{T} \mathbf{U}\right]_{j k}=\frac{1}{N} \sum_{t=\tau+1}^{N} u(t) u(t-\tau), \quad \tau=|j-k| .
$$

The asymptotic (large $N$ ) covariance matrix of the least squares estimate (5) is

$$
\begin{aligned}
\operatorname{AsCov}\{\hat{\mathbf{g}}\} & =\mathbf{P}, \quad \mathbf{P}=\frac{\lambda_{e}}{N} \mathbf{R}^{-1}, \\
\mathbf{R}_{j k} & =\overline{\mathrm{E}}\{u(t) u(t-\tau)\}, \quad \tau=|j-k|,
\end{aligned}
$$

where $\bar{E}$ denotes quasi-stationary expectation, defined in [6]. For a stationary zero mean stochastic process this equals ordinary expectation and $r_{\tau}=\mathrm{E}\{u(t) u(t-\tau)\}$ is the covariance function. We use As to denote variance results that are asymptotic in the number of data points, $N$.

We will study functions of the estimated impulse response vector, and, in particular, the frequency response estimate

$$
\hat{G}\left(e^{i \omega}\right)=\Gamma^{*}\left(e^{i \omega}\right) \hat{\mathbf{g}}, \quad \Gamma\left(e^{i \omega}\right)=\left[1, e^{i \omega}, \ldots, e^{i n \omega}\right]^{T},
$$

where super-index $*$ means conjugate transpose. We use $G_{o}\left(e^{i \omega}\right)=\Gamma^{*}\left(e^{i \omega}\right) \mathbf{g}_{o}$ for the true transfer function. The analysis will be based on first order Taylor series expansions and is therefore valid in the small error regime, that is asymptotically for large $N$. The definition $\operatorname{Cov}\{Z\}=\mathrm{E}\left\{Z Z^{*}\right\}$ is used for zero mean complex vector-valued random variables. The error variances of the frequency response estimate $\hat{G}\left(e^{i \omega}\right)=\Gamma^{*}\left(e^{i \omega}\right) \hat{\mathbf{g}}$, with

$$
\Delta G\left(e^{i \omega}\right)=\hat{G}\left(e^{i \omega}\right)-G_{o}\left(e^{i \omega}\right)=\Gamma^{*}\left(e^{i \omega}\right) \Delta \mathbf{g},
$$

are

$$
\begin{aligned}
\operatorname{Var}\left\{\hat{G}\left(e^{i \omega}\right)\right\} & =\Gamma^{*}\left(e^{i \omega}\right) \mathbf{P}_{N} \Gamma\left(e^{i \omega}\right), \\
\operatorname{As} \operatorname{Var}\left\{\hat{G}\left(e^{i \omega}\right)\right\} & =\Gamma^{*}\left(e^{i \omega}\right) \mathbf{P} \Gamma\left(e^{i \omega}\right) .
\end{aligned}
$$

Using $\left|G\left(e^{i \omega}\right)\right|^{2}=G\left(e^{i \omega}\right) G^{*}\left(e^{i \omega}\right)$, a first order perturbation analysis gives

$$
\Delta\left|G\left(e^{i \omega}\right)\right|^{2} \approx \Delta G\left(e^{i \omega}\right) G_{o}^{*}\left(e^{i \omega}\right)+G_{o}\left(e^{i \omega}\right) \Delta G^{*}\left(e^{i \omega}\right) .
$$

Now (11) gives ${ }^{1}$

$$
\Delta\left|G\left(e^{i \omega}\right)\right|^{2} \approx 2\left[\operatorname{Re}\left\{G_{o}\left(e^{i \omega}\right) \Gamma\left(e^{i \omega}\right)\right\}\right]^{T} \Delta \mathbf{g},
$$

and thus

$$
\begin{aligned}
& \text { AsVar }\left\{\left|\hat{G}\left(e^{i \omega}\right)\right|^{2}\right\}= \\
& 4\left[\operatorname{Re}\left\{G_{o}\left(e^{i \omega}\right) \Gamma\left(e^{i \omega}\right)\right\}\right]^{T} \mathbf{P}\left[\operatorname{Re}\left\{G_{o}\left(e^{i \omega}\right) \Gamma\left(e^{i \omega}\right)\right\}\right]
\end{aligned}
$$

Define

$$
\begin{array}{cc}
\omega_{p}^{o}=\arg \max _{\omega}\left|G_{o}\left(e^{i \omega}\right)\right|^{2}, & \beta_{o}=\left|G_{o}\left(e^{i \omega_{p}^{o}}\right)\right|^{2}, \\
\hat{\omega}_{p}=\arg \max _{\omega}\left|\hat{G}\left(e^{i \omega}\right)\right|^{2}, & \hat{\beta}=\left|\hat{G}\left(e^{i \hat{\omega}_{p}}\right)\right|^{2}, \\
\Delta \omega_{p}=\hat{\omega}_{p}-\omega_{p}^{o}, & \Delta \beta=\hat{\beta}-\beta_{o} .
\end{array}
$$

The parameter $\beta_{o}$ is the squared $\mathscr{H}_{\infty}$-norm of $G_{o}\left(e^{i \omega}\right)$, and $\hat{\beta}$ is a corresponding estimate.

Proposition 1: The asymptotic error variance of the least squares based estimate of the squared $\mathscr{H}_{\infty}$-norm of $G_{o}\left(e^{i \omega}\right)$, defined by (14), equals

$$
\begin{aligned}
& \operatorname{As} \operatorname{Var}\{\hat{\beta}\}= \\
& 4\left[\operatorname{Re}\left\{G_{o}\left(e^{i \omega_{p}^{o}}\right) \Gamma\left(e^{i \omega_{p}^{o}}\right)\right\}\right]^{T} \mathbf{P}\left[\operatorname{Re}\left\{G_{o}\left(e^{i \omega_{p}^{o}}\right) \Gamma\left(e^{i \omega_{p}^{o}}\right)\right\}\right] .
\end{aligned}
$$

Proof: A first order perturbation analysis gives

$$
\Delta \beta \approx\left[\left.\frac{\partial\left|G_{o}\left(e^{i \omega}\right)\right|^{2}}{\partial \omega}\right|_{\omega=\omega_{p}^{o}}\right] \Delta \omega_{p}+\left[\left.\frac{\partial\left|G\left(e^{i \omega_{p}^{o}}\right)\right|^{2}}{\partial \mathbf{g}}\right|_{\mathbf{g}=\mathbf{g}_{0}}\right]^{T} \Delta \mathbf{g},
$$

where the first term of the right hand side is zero since $\omega_{p}^{o}$ is a critical point (maximum). The variance of second term is $\operatorname{As} \operatorname{Var}\left\{\left|\hat{G}\left(e^{i \omega_{p}^{o}}\right)\right|^{2}\right\}$, which is given by (13).

This result implies that there is no asymptotic variance cost due to uncertainty in frequency since the corresponding derivative is zero at $\omega_{p}^{o}$.

\section{Optimal InPUt Design: A First ORDER FIR EXAMPLE}

Consider the first order FIR system

$$
y(t)=g_{0} u(t)+g_{1} u(t-1)+e(t),
$$

for which (9) equals

$$
\mathbf{R}=\left[\begin{array}{ll}
r_{0} & r_{1} \\
r_{1} & r_{0}
\end{array}\right]
$$

Here $\left.r_{\tau}=\overline{\mathrm{E}}\{u(t) u(t-\tau))\right\}, \tau=0,1$, are covariances of a quasi-stationary process. Note that $r_{0} \geq\left|r_{1}\right|$ gives $\mathbf{R} \geq 0$. Variance expression (8) equals

$$
\operatorname{AsCov}\left\{\left[\begin{array}{l}
\hat{g}_{0} \\
\hat{g}_{1}
\end{array}\right]\right\}=\frac{\lambda_{e}}{N} \mathbf{R}^{-1} .
$$

We will consider linear functions of the impulse coefficients $\eta=c_{1}^{*} g_{0}+c_{1}^{*} g_{1}=\mathbf{c}^{*} \mathbf{g}$, where $\mathbf{c}=\left[\begin{array}{ll}c_{1} & c_{2}\end{array}\right]^{T}$ and $\mathbf{g}=\left[\begin{array}{ll}g_{0} & g_{1}\end{array}\right]^{T}$, with the special case of frequency response estimation, $\mathbf{c}=\left[1 e^{i \omega}\right]^{t}$. We allow $\mathbf{R}$ later on in this section to be rank deficient as long as the asymptotic variance of the least squares estimate of scalar parameter $\eta$ is finite. This is the case if $\mathbf{c}$ belongs to the range space of $\mathbf{R}$. Then

\footnotetext{
${ }^{1} \operatorname{Re}\{z\}$ is the real part and $\operatorname{Im}\{z\}$ is the imaginary part of a complex
} number $z$. 
$\operatorname{As} \operatorname{Var}\{\hat{\eta}\}=\mathbf{c}^{*} \mathbf{R}^{\dagger} \mathbf{c}$, where $\dagger$ denotes any pseudo inverse, c.f. [13].

Consider the optimization problem ${ }^{2}$

$$
\begin{array}{ll}
\mathrm{P} 1: & \min _{0} \mathbf{c}^{*} \mathbf{R}^{-1} \mathbf{c} \\
\text { s.t. } & r_{0} \leq \gamma, \quad \mathbf{R} \geq 0 .
\end{array}
$$

The objective is to minimize the variance of $\hat{\eta}=\mathbf{c}^{*} \hat{\mathbf{g}}$ subject to a bound on the variance of the input signal. The optimal solution will satisfy $r_{0}=\gamma$. As shown in [10] this is closely related to the least costly identification optimization problem, [1],

$$
\begin{array}{ll}
\mathrm{P} 2: & \min _{r_{0}, r_{1}} r_{0} \\
\text { s.t. } & \mathbf{c}^{*} \mathbf{R}^{-1} \mathbf{c} \leq 1, \quad \mathbf{R} \geq 0 .
\end{array}
$$

Optimization problem $\mathrm{P} 2$ aims to find the minimum power input signal for which the normalized variance of $\mathbf{c}^{*} \hat{\mathbf{g}}$ is smaller than or equal to one. It is possible to re-scale P2 to allow for other variance values. Also scaling the solution of $\mathrm{P} 2$ to obtain $r_{0}=\gamma$ gives the solution to problem P1, see [10].

Problem P2 is equivalent to the optimization problem

$$
\begin{array}{ll}
\text { P3 : } & \min _{r_{0}, r_{1}} r_{0} \\
\text { s.t. } & \mathbf{R} \geq \mathbf{c c}^{*} .
\end{array}
$$

For completeness a proof is given in Appendix A. Notice that (19) implies $\mathbf{R} \geq 0$. Problem P3 is a Semi-Definite Program (SDP), since the cost and the constraint in (19) are linear in $r_{0}$ and $r_{1}$. This observation is the key to obtain the following analytic result.

Proposition 2: The optimal solution to $\mathrm{P} 2$ and $\mathrm{P} 3$ is $^{3}$

$$
\begin{aligned}
r_{0}^{\star} & =\frac{\left|c_{1}\right|^{2}+\left|c_{2}\right|^{2}+\sqrt{\left(\left|c_{1}\right|^{2}-\left|c_{2}\right|^{2}\right)^{2}+4\left[\operatorname{Im}\left\{c_{1} c_{2}^{*}\right\}\right]^{2}}}{2} \\
r_{1}^{\star} & =\operatorname{Re}\left\{c_{1} c_{2}^{*}\right\},
\end{aligned}
$$

which simplifies to

$$
r_{0}^{\star}=\max \left\{c_{1}^{2}, c_{2}^{2}\right\}, \quad r_{1}^{\star}=c_{1} c_{2}
$$

for a real-valued vector $\mathbf{c}$.

The proof is given in Appendix B.

There are many ways to realize a stochastic signal with given $r_{0}$ and $r_{1}$. If $r_{0}>\left|r_{1}\right|$ the AR process

$u(t)=-a u(t-1)+e_{u}(t), \quad a=-\frac{r_{1}}{r_{0}}, \mathrm{E}\left\{e_{u}^{2}(t)\right\}=\left(1-a^{2}\right) r_{0}$

can be used. If $r_{0}=\left|r_{1}\right|$ we need to use a sinusoidal signal. We will compare the optimal solution (20) to the case when the sinusoidal signal $u(t)=C \cos (\bar{\omega} t)$ is used as input in the experiment. The quasi-stationary covariance function for a sinusoidal signal, see [6], is

$$
\begin{aligned}
& r_{0}=\left\{\begin{array}{ll}
C^{2}, & \bar{\omega}=0, \pi \\
\frac{C^{2}}{2}, & \text { otherwise }
\end{array},\right. \\
& r_{k}=r_{0} \cos (\bar{\omega} k), k \geq 1 .
\end{aligned}
$$

${ }^{2}$ For matrices $X \geq Y$ means that $[X-Y]$ is a positive semi-definite matrix. ${ }^{3}$ We use the convention $\sqrt{x^{2}}=|x| \geq 0$.
For a sinusoidal signal with frequency $\bar{\omega}(\bar{\omega} \neq 0, \pi)$ the variance constraint

$$
\mathbf{c}^{*} \mathbf{R}^{-1} \mathbf{c}=\frac{1}{r_{0}} \frac{c_{1} c_{1}^{*}+c_{2} c_{2}^{*}-\left(c_{1} c_{2}^{*}+c_{1}^{*} c_{2}\right) \cos (\bar{\omega})}{1-\cos ^{2}(\bar{\omega})}=1
$$

implies that the corresponding covariances must be equal to (we use an over-bar to indicate this normalized variance property)

$$
\begin{aligned}
& \bar{r}_{0}=\frac{\left|c_{1}\right|^{2}+\left|c_{2}\right|^{2}-2 \operatorname{Re}\left\{c_{1} c_{2}^{*}\right\} \cos (\bar{\omega})}{1-\cos ^{2}(\bar{\omega})}, \\
& \bar{r}_{1}=\bar{r}_{0} \cos (\bar{\omega}) .
\end{aligned}
$$

If $\bar{\omega}=0$ the matrix $\mathbf{R}$ will be singular and in order to obtain a well defined estimation problem we must constrain the cvector to satisfy $c_{1}=c_{2}=c$. If $\bar{\omega}=\pi$ we require that $c_{1}=$ $-c_{2}=c$. The solutions to $\mathbf{c}^{*} \mathbf{R}^{\dagger} \mathbf{c}=1$ for these two special cases are

$$
\bar{r}_{0}=c^{2}, \quad \bar{r}_{1}=c^{2} \cos (\bar{\omega}), \quad \bar{\omega}=0, \pi .
$$

We will now solve the optimal input problem $\mathrm{P} 3$ for specific choices of $\mathbf{c}$ :

Special Case 1: The simplest example is $\operatorname{As} \operatorname{Var}\left\{\hat{g}_{k}\right\}$, $k=0,1$, for which $\mathbf{c}=\left[\begin{array}{ll}1 & 0\end{array}\right]^{T}$ and $\mathbf{c}=\left[\begin{array}{ll}0 & 1\end{array}\right]^{T}$. The optimal solutions for both cases are $r_{0}^{\star}=1$ and $r_{1}^{\star}=0$, which can be realized by a white noise process or a sinusoidal signal with $\bar{\omega}=\pi / 2$.

Special Case 2: The next special case is for the frequency response estimate $\hat{G}\left(e^{i \omega}\right),(12)$, where $\mathbf{c}=\left[1 e^{i \omega}\right]^{T}$ and

$\left|c_{1}\right|^{2}=\left|c_{2}\right|^{2}=1, \operatorname{Re}\left\{c_{1} c_{2}^{*}\right\}=\cos (\omega), \operatorname{Im}\left\{c_{1} c_{2}^{*}\right\}=-\sin (\omega)$.

The optimal solution (20) equals

$$
r_{0}^{\star}=1+|\sin (\omega)|, \quad r_{1}^{\star}=\cos (\omega) .
$$

We will compare the optimal solution (24) with a sinusoidal input signal with frequency $\bar{\omega}=\omega \neq 0, \pi$. This means that we excite the system exactly at the frequency specified by $G\left(e^{i \omega}\right)$. The sinusoidal solution (23) equals

$$
\bar{r}_{0}=2, \quad \bar{r}_{1}=2 \cos (\omega) .
$$

For $\omega=\pi / 2$ the optimal solution and the sinusoidal solution have identical powers and correlations at lag 1, but for other frequencies the optimal solution is up to a factor of two more power efficient than the sinusoidal input with frequency $\bar{\omega}=\omega$. If we instead take the frequency, $\bar{\omega}$, of the sinusoidal input signal such that

$$
\cos (\bar{\omega})=\frac{\cos (\omega)}{1+|\sin (\omega)|} \quad \Rightarrow \quad \bar{r}_{0}=r_{0}^{\star}, \bar{r}_{1}=r_{1}^{\star},
$$

i.e., this choice of frequency corresponds to one way to realize the optimal input signal given by (24). For $0<$ $\omega<\pi / 2$, Equation (26) implies that $\bar{\omega}>\omega$. Hence, here it is more power efficient to use a sinusoidal signal with frequency higher than $\omega$.

For both $\omega=0$ and $\omega=\pi$, the sinusoidal solution (23) equals the optimal solution $r_{0}^{\star}=1$ and $r_{1}^{\star}=\cos (\omega)$.

Special Case 3: The final special case we want to analyze is for the amplitude frequency function estimate $\left|\hat{G}\left(e^{i \omega}\right)\right|^{2}$, with

$$
\begin{aligned}
\mathbf{c} & =2 \operatorname{Re}\left\{\left[G\left(e^{i \omega}\right), G\left(e^{i \omega}\right) e^{i \omega}\right]\right\}^{T} \\
& =2\left[g_{0}+g_{1} \cos (\omega), g_{0} \cos (\omega)+g_{1}\right]^{T} .
\end{aligned}
$$


Hence, the optimal input (21) has

$$
\begin{aligned}
& r_{0}^{\star}=4 \max \left\{\left(g_{0}+g_{1} \cos (\omega)\right)^{2},\left(g_{0} \cos (\omega)+g_{1}\right)^{2}\right\}, \\
& r_{1}^{\star}=4\left[g_{0}+g_{1} \cos (\omega)\right]\left[g_{0} \cos (\omega)+g_{1}\right] .
\end{aligned}
$$

The sinusoidal input solution (23) with $\bar{\omega}=\omega$ has

$$
\begin{aligned}
& \bar{r}_{0}=4\left(g_{0}+g_{1} \cos (\omega)\right)^{2}+g_{1}^{2} \sin ^{2}(\omega)=4\left|G\left(e^{i \omega}\right)\right|^{2}, \\
& \bar{r}_{1}=4\left|G\left(e^{i \omega}\right)\right|^{2} \cos (\omega) .
\end{aligned}
$$

It is interesting to note that

$$
\bar{r}_{0}-r_{0}^{\star}=4 \min \left\{g_{0}^{2}, g_{1}^{2}\right\} \sin ^{2}(\omega) .
$$

Hence, the sinusoidal input is close to optimal for low and high frequencies. It is optimal for $\omega=0, \pi$.

The $\mathscr{H}_{\infty}$-norm problem (14) is easy to solve for a first order FIR system. Since

$$
\left|G\left(e^{i \omega}\right)\right|^{2}=g_{0}^{2}+g_{1}^{2}+2 g_{0} g_{1} \cos (\omega),
$$

we have that $\omega_{p}=0$ if $g_{0} g_{1}>0$ and $\omega_{p}=\pi$ if $g_{0} g_{1}<0$. Proposition 1 implies that we just can reuse the previous result for $\operatorname{As} \operatorname{Var}\left\{\left|\hat{G}\left(e^{i \omega_{p}^{o}}\right)\right|^{2}\right\}$. It is interesting to note that $\omega=0$ and $\omega=\pi$ are two cases when the optimal solution coincides with the sinusoidal solution with $\bar{\omega}=\omega$. For the special case $g_{0} g_{1}=0$ the gain $\left|G\left(e^{i \omega}\right)\right|$ does not depend on frequency and $\omega_{p}$ is not defined. However, the gain is constant and the variance of the gain estimate only depends on the power of the input signal.

\section{General RESUlts}

\section{A. Optimal Input Problem}

The sequence $\left\{r_{0}, r_{1}, \ldots r_{n}\right\}$ is the initial part of a covariance sequence of a stationary stochastic process if the corresponding symmetric Toeplitz matrix with the sequence as first column is positive definite, $\mathbf{R}>0$. By using the less restrictive condition $\mathbf{R} \geq 0$ we will, however, allow for rank deficient solutions. In this case the Carathéodory parametrization of a covariance matrix, see [14], can be used to find a time realization of the input signal $u(t)$. Such a realization is based on sinusoidal signals. It is also possible to perturb the optimal rank deficient solution by slightly increasing $r_{0}$. This corresponds to adding white noise to the Carathéodory solution, and is a way to robustify the optimal solution.

Reconsider the optimization problem

$$
\begin{aligned}
& \mathrm{P} 3: \min _{\mathbf{r}} r_{0} \\
& \text { s.t. } \mathbf{R} \geq \mathbf{c c}^{*} .
\end{aligned}
$$

where $\mathbf{c}=\left[\begin{array}{llll}c_{1} & c_{2}, \ldots c_{n+1}\end{array}\right]^{T}$ is a given column vector, $\mathbf{r}=$ $\left[r_{0}, r_{1}, \ldots r_{n}\right]^{T}$ and $\mathbf{R}$ is the symmetric Toeplitz matrix with first column $\mathbf{r}$. An optimal solution to P3 will be denoted by $r^{\star}$.

We are not aware of a general analytic solution of (27) for $n \geq 2$, but P3 is a convex optimization problem (SemiDefinite Program/SDP) for which excellent numerical methods exist. We have used the MATLAB toolbox YALMIP with the LMI Lab solver in the simulation study, [5].

It is, however, possible to obtain more insight in the frequency response input optimization problem with $\mathbf{c}=\Gamma\left(e^{i \omega}\right)$ defined by (10). From [8], $\Gamma^{*}\left(e^{i \omega}\right) \mathbf{R}^{-1} \Gamma\left(e^{i \omega}\right) \approx n / \Phi_{u}(\omega)$, where $\Phi_{u}(\omega)$ is the power spectral density of $u(t)$. Hence, the optimization problem (27) can be approximated as

$$
\begin{aligned}
& \min _{\mathbf{r}} \frac{1}{2 \pi} \int_{-\pi}^{\pi} \Phi_{u}(\bar{\omega}) d \bar{\omega} \\
& \text { s.t. } \Phi_{u}(\omega) \geq n .
\end{aligned}
$$

Note that the constraint is only at the frequency of interest $\omega$. This indicates that the optimal signal should have a line spectrum in order to minimize the integral, and at the same time satisfy the power density condition at frequency $\omega$. This means that the optimal solution for large $n$ would be a sinusoidal signal.

\section{B. Sinusoidal Input}

We would again like to compare the optimal solution of (19) to using a sinusoidal signal $u(t)=C \cos (\bar{\omega} t)$. The corresponding quasi-stationary covariance function $\bar{r}_{k}$ is given by (22), which leads to

$$
\mathbf{R}=r_{0} \operatorname{Re}\left\{\Gamma\left(e^{i \bar{\omega}}\right) \Gamma^{*}\left(e^{i \bar{\omega}}\right)\right\}, \Gamma\left(e^{i \bar{\omega}}\right)=\left[1, e^{i \bar{\omega}}, \ldots e^{i n \bar{\omega}}\right]^{T}
$$

where $r_{0}=C^{2}$ for $\bar{\omega}=0, \pi$ and otherwise $r_{0}=C^{2} / 2$. For $n \geq 0$ the rank of the matrix $\mathbf{R}$ is one for $\bar{\omega}=0, \pi$ and otherwise two. Therefore it is only possible to estimate a function of the frequency response $G\left(e^{i \bar{\omega}}\right)$ at $\omega=\bar{\omega}$. We need to use a pseudo-inverse of $\mathbf{R}$ in the frequency response variance expression (12),

$\operatorname{As} \operatorname{Var}\left\{\hat{G}\left(e^{i \omega}\right)\right\}=\frac{\lambda_{e}}{N r_{0}} \Gamma^{*}\left(e^{i \omega}\right)\left[\operatorname{Re}\left\{\Gamma\left(e^{i \omega}\right) \Gamma^{*}\left(e^{i \omega}\right)\right\}\right]^{\dagger} \Gamma\left(e^{i \omega}\right)$

Now AsVar $\left\{\hat{G}\left(e^{-i \omega}\right)\right\}=\operatorname{As} \operatorname{Var}\left\{\hat{G}\left(e^{i \omega}\right)\right\}$ since the impulse response is real valued, and hence

$$
\begin{aligned}
& \operatorname{As} \operatorname{Var}\left\{\hat{G}\left(e^{i \omega}\right)\right\}= \\
& \frac{\lambda_{e}}{N r_{0}} \frac{1}{2} \operatorname{Trace}\left\{\Gamma^{*}\left(e^{i \omega}\right)\left[\operatorname{Re}\left\{\Gamma\left(e^{i \omega}\right) \Gamma^{*}\left(e^{i \omega}\right)\right\}\right]^{\dagger} \Gamma\left(e^{i \omega}\right)\right. \\
& \left.+\Gamma^{*}\left(e^{-i \omega}\right)\left[\operatorname{Re}\left\{\Gamma\left(e^{-i \omega}\right) \Gamma^{*}\left(e^{-i \omega}\right)\right\}\right]^{\dagger} \Gamma\left(e^{-i \omega}\right)\right\} \\
& =\frac{\lambda_{e}}{N r_{0}} \operatorname{Trace}\left\{\left[\operatorname{Re}\left\{\Gamma\left(e^{i \omega}\right) \Gamma^{*}\left(e^{i \omega}\right)\right\}\right]^{\dagger}\left[\operatorname{Re}\left\{\Gamma\left(e^{i \omega}\right) \Gamma^{*}\left(e^{i \omega}\right)\right\}\right]\right\}
\end{aligned}
$$

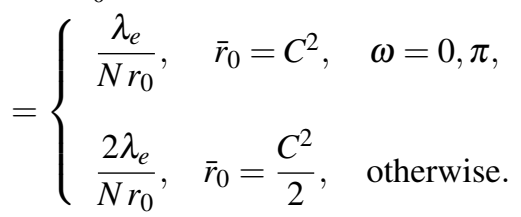

Here we have used the fact that $\operatorname{Trace}\left\{\mathbf{R}^{\dagger} \mathbf{R}\right\}=\operatorname{rank}\{\mathbf{R}\}$ and that the rank of $\mathbf{R}$ for the sinusoidal case is one or two, depending on the frequency.

Expression (30) is identical to asymptotic variance of the Empirical Transfer Function Estimate (ETFE), see [6]. The same expression is also obtained in [3] for periodic input signals with $\mathbf{R}$ having full rank. The derivation is, however, different. 


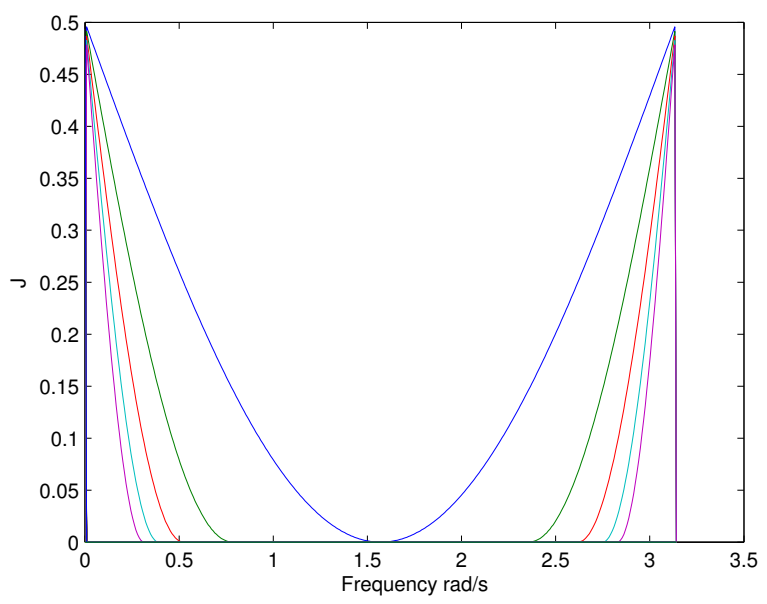

Fig. 1. Performance indices, $J(n)$, for frequency response estimation, $n=$ $1, \ldots, 5 . J(n)$ is a monotonically decreasing function of $n$. The upper (blue) curve is for $n=1$ and the lower curve (magenta) is for $n=5$.

Next consider (13) with $\bar{\omega}=\omega$,

$$
\begin{aligned}
& \text { AsVar }\left\{\left|\hat{G}\left(e^{i \omega}\right)\right|^{2}\right\}= \\
& \frac{4 \lambda_{e}}{N}\left[\operatorname{Re}\left\{G_{o}^{*}\left(e^{i \omega}\right) \Gamma^{*}\left(e^{i \omega}\right)\right\}\right] \mathbf{R}^{\dagger}\left[\operatorname{Re}\left\{G_{o}^{*}\left(e^{i \omega}\right) \Gamma^{*}\left(e^{i \omega}\right)\right\}\right]^{T} \\
& =\frac{4 \lambda_{e}}{N r_{0}} \mathbf{g}_{o}^{T}\left[\operatorname{Re}\left\{\Gamma\left(e^{i \omega}\right) \Gamma^{*}\left(e^{i \omega}\right)\right\}\right]\left[\operatorname{Re}\left\{\Gamma\left(e^{i \omega}\right) \Gamma^{*}\left(e^{i \omega}\right)\right\}\right]^{\dagger} \\
& \times\left[\operatorname{Re}\left\{\Gamma\left(e^{i \omega}\right) \Gamma^{*}\left(e^{i \omega}\right)\right\}\right] \mathbf{g}_{o}=\frac{4 \lambda_{e}}{N r_{0}} \mathbf{g}_{o}^{T}\left[\operatorname{Re}\left\{\Gamma\left(e^{i \omega}\right) \Gamma^{*}\left(e^{i \omega}\right)\right\}\right] \mathbf{g}_{o} \\
& =\frac{4 \lambda_{e}}{N r_{0}}\left|G_{o}\left(e^{i \omega}\right)\right|^{2} .
\end{aligned}
$$

Hence, we also have a simple and intuitive variance expression for $\operatorname{As} \operatorname{Var}\left\{\left|\hat{G}\left(e^{i \omega}\right)\right|^{2}\right\}$. Note that $r_{0}$ is the power of the sinusoidal signal, and $\lambda_{e} / r_{0}$ is the noise to signal ratio. The variance is proportional to the squared amplitude, $\left|G_{o}\left(e^{i \omega}\right)\right|^{2}$, of the frequency response function.

\section{NUMERICAL STUDY}

We will study random $n$ :th FIR systems, with normally distributed coefficients, $g_{k} \sim \mathscr{N}(0,1)$, with $\lambda_{e}=1$ and numerically solve problem P3, (27), for the optimal solution $\mathbf{r}^{\star}$. The performance index

$$
J=\frac{\bar{r}_{0}-r_{0}^{\star}}{\bar{r}_{0}}
$$

is used to measure how much power reduction is gained by using the optimal solution compared to a sinusoidal input with frequency $\omega$. Here $\bar{r}_{0}$ is the power of a sinusoidal input signal with frequency $\omega$ that gives the same estimation error variance as the optimal input signal.

We will start by studying the frequency response estimation problem with $\mathbf{c}=\Gamma\left(e^{i \omega}\right)$ defined by (10) for systems of orders 1 to 5. The optimal input problem (27) is solved using MATLAB/LMI LAB/YALMIP, [5], for $\omega_{k}=k \pi / 360$, $k=0, \ldots, 360$. The corresponding performance indices are plotted in Figure 1. For $n=1$ we have recovered the results (24) and (25), i.e., $J(1)=[1-\sin (\omega)] / 2$. We note that the sinusoidal signal is optimal for an increasing range

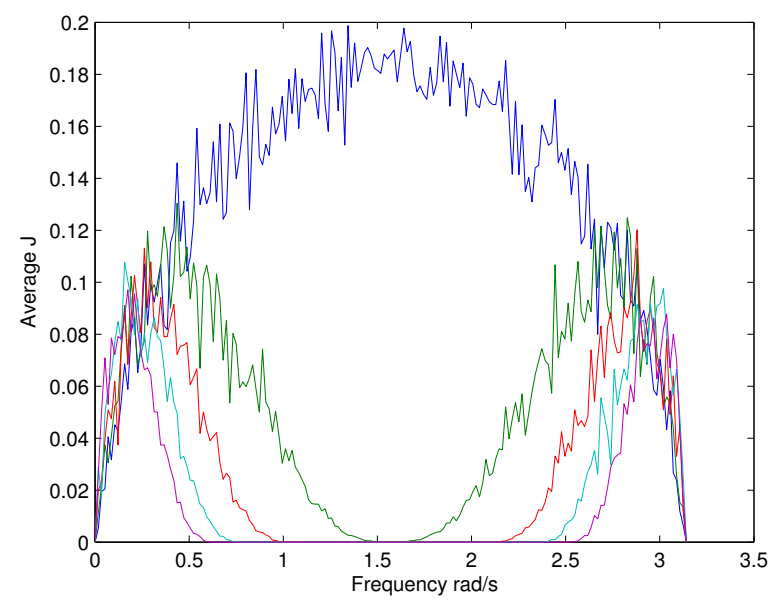

Fig. 2. Average performance indices, $J(n)$, for amplitude frequency response estimation, $n=1, \ldots, 5$. The upper (blue) curve is for $n=1$ and the lower curve (magenta) is for $n=5$.

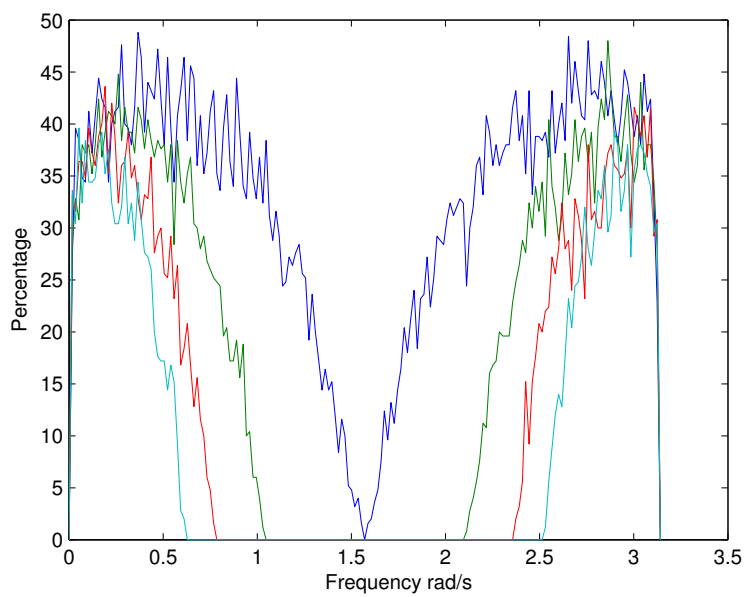

Fig. 3. Percentage of systems where the optimal solution is not a sinusoidal signal for amplitude frequency response estimation. The upper (blue) curve is for $n=2$ and the lower curve (cyan) is for $n=5$. For $n=1$ we have $100 \%$ for all frequencies except 0 and $\pi$.

of medium frequencies as $n$ increases, while at low and high frequencies we gain almost a factor of two in power reduction by using the optimal input signal.

Next we will study the amplitude frequency function, $\left|G\left(e^{\omega}\right)\right|^{2}$, case for specified frequency $\omega$. Figure 2 shows the the average performance index function $J(n)$, at the frequencies $\omega_{k}=k \pi / 180, k=0, \ldots, 180$, over $250 n$ :th order random FIR systems, $n=1$ to 5 . Figure 3 shows the percentage of systems for which the optimal solution is not a sinusoidal signal. As $n$ increase the optimal solution is more often a sinusoidal signal. Also notice that the gain in power reduction when the solution is not a sinusoidal signal is higher than given in Figure 2, since the average performance index function $J(n)$ also includes all cases when $J=0$.

The conclusions from this example are that there is a lot to be gained in terms of power reduction for lower and higher frequencies, but less for frequencies around $\pi / 2$. However, as 
the model order increases a sinusoidal signal with frequency $\omega$ becomes more often optimal for estimating $G\left(e^{\omega}\right)$ or $\left|G\left(e^{\omega}\right)\right|^{2}$.

We have also done extensive simulations studies for the $\mathscr{H}_{\infty}$-norm case, where the peak frequency $\omega_{p}$ is found by numerical optimization for random systems. The observation is that in all performed simulation examples a sinusoidal signal with frequency equal to the peak frequency is optimal for estimating the $\mathscr{H}_{\infty}$-norm. That this is indeed the case has recently been proved in [16].

\section{CONCLUSION}

The objective of this contribution has been to study optimal input design for frequency response estimation. For lower order systems up to fifty percent in power reduction can be gained by using an optimal input signal, but for higher order systems a sinusoidal signal is a good choice. A sinusoidal signal with frequency $\omega$ is quite good for estimating $G\left(e^{i \omega}\right)$, but gives little information about other system properties. For lower order systems the parameter structure can be used to obtain even better information about $G\left(e^{i \omega}\right)$. For higher order systems this structural information is of less importance and the optimal input just focuses on $G\left(e^{i \omega}\right)$

An important problem in the robust control applications is the estimation of $\mathscr{H}_{\infty}$-norm of a linear system. The problem is almost trivial in the case we know the peak frequency, but we have shown that errors in the frequency estimate have a small effect due to the maximum property. In [16] it is shown that the optimal input signal is a sinusoidal signal frequency equal to the peak frequency. Iterative methods for input design for $\mathscr{H}_{\infty}$ estimation are discussed in [15].

We have only studied the asymptotic case of $N \gg 1$, but variance expression (6) allows for finite sample results. The time realization problem can then be approached by finding an input sequence $\mathbf{u}$ from

$$
\min _{\mathbf{u}}\left\|\frac{1}{N} \mathbf{U}^{T} \mathbf{U}-\mathbf{R}^{\star}\right\|_{F}
$$

where $\mathbf{U}$ is defined as in (4) and $\mathbf{R}^{\star}$ is the Toeplitz matrix constructed from the optimal solution to a corresponding input optimization problem. Recall from (7) that $\mathbf{U}^{T} \mathbf{U} / N$ has a Toeplitz structure associated with the covariance function of a moving-average process of order $N$. Such problems can be solved using iterative methods, see [12].

\section{APPENDIX}

\section{A. Equivalence of (18) and (19)}

The equivalence of optimization problem (18) and (19) can be proved by studying the matrix inequality

$$
\left[\begin{array}{ll}
\mathbf{R} & \mathbf{c} \\
\mathbf{c}^{*} & 1
\end{array}\right] \geq 0
$$

Recall that we assume $\mathbf{R} \geq 0$. Then

$$
\left[\begin{array}{cc}
\mathbf{R}^{-1} & 0 \\
-\mathbf{c}^{*} \mathbf{R}^{-1} & 1
\end{array}\right]\left[\begin{array}{cc}
\mathbf{R} & \mathbf{c} \\
\mathbf{c}^{*} & 1
\end{array}\right]\left[\begin{array}{cc}
\mathbf{R}^{-1} & -\mathbf{R}^{-1} \mathbf{c} \\
0 & 1
\end{array}\right]=\left[\begin{array}{cc}
\mathbf{R}^{-1} & 0 \\
0 & 1-\mathbf{c}^{*} \mathbf{R}^{-1} \mathbf{c}
\end{array}\right] \geq 0
$$

and

$$
\left[\begin{array}{cc}
\mathbf{I} & -\mathbf{c} \\
0 & 1
\end{array}\right]\left[\begin{array}{ll}
\mathbf{R} & \mathbf{c} \\
\mathbf{c}^{*} & 1
\end{array}\right]\left[\begin{array}{cc}
\mathbf{I} & 0 \\
-\mathbf{c}^{*} & 1
\end{array}\right]=\left[\begin{array}{cc}
\mathbf{R}-\mathbf{c c}^{*} & 0 \\
0 & 1
\end{array}\right] \geq 0
$$

which shows the claim. If $\mathbf{R}$ is singular then $c$ must be in the range space of $\mathbf{R}$ and the pseudo-inverse must be used in the above calculations.

\section{B. Proof of (20)}

The matrix

$$
\mathbf{R}-\mathbf{c c}^{*}=\left[\begin{array}{ll}
r_{0}-\left|c_{1}\right|^{2} & r_{1}-c_{1} c_{2}^{*} \\
r_{1}-c_{1}^{*} c_{2} & r_{0}-\left|c_{2}\right|^{2}
\end{array}\right],
$$

is positive definite if and only if the following determinant conditions hold

$$
\begin{aligned}
& r_{0}-\left|c_{1}\right|^{2} \geq 0, \quad r_{0}-\left|c_{2}\right|^{2} \geq 0 \\
& \left(r_{0}-\left|c_{1}\right|^{2}\right)\left(r_{0}-\left|c_{2}\right|^{2}\right)-\left(r_{1}-c_{1} c_{2}^{*}\right)\left(r_{1}-c_{1}^{*} c_{2}\right) \geq 0 .
\end{aligned}
$$

The second part of (33)

$$
-\left(r_{1}-c_{1} c_{2}^{*}\right)\left(r_{1}-c_{1}^{*} c_{2}\right)=-\left|r_{1}-c_{1} c_{2}^{*}\right|^{2},
$$

has minimum $-\left[\operatorname{Im}\left\{c_{1} c_{2}^{*}\right\}\right]^{2}$ for $r_{1}=r_{1}^{\star}=\operatorname{Re}\left\{c_{1} c_{2}^{*}\right\}$. This gives the least restrictive version of (33)

$$
\left(r_{0}-\left|c_{1}\right|^{2}\right)\left(r_{0}-\left|c_{2}\right|^{2}\right) \geq\left[\operatorname{Im}\left\{c_{1} c_{2}^{*}\right\}\right]^{2} .
$$

The second order equation

$$
\left(r_{0}-\left|c_{1}\right|^{2}\right)\left(r_{0}-\left|c_{2}\right|^{2}\right)=\left[\operatorname{Im}\left\{c_{1} c_{2}^{*}\right\}\right]^{2}
$$

has one solution that is larger than or equal to $\max \left\{\left|c_{1}\right|^{2},\left|c_{2}\right|^{2}\right\}$ and one that is smaller than or equal to $\min \left\{c_{1} c_{2}^{*}, c_{2} c_{2}^{*}\right\}$. Only the larger solution will satisfy the first constraints (32). Solving the second order equation gives that covariance function of the optimal input signal is given by (20), which simplifies to (21) for the real valued case.

\section{REFERENCES}

[1] X. Bombois, G. Scorletti, M. Gevers, P. M. J. Van den Hof, and R. Hildebrand. Least costly identification experiment for control. Automatica, 42(10):1651-1662, 2006.

[2] H. Hjalmarsson. System identification of complex and structured systems. European Journal of Control, pages 275-231, 2009.

[3] H. Hjalmarsson and B. Ninness. Least-squares estimation of a class of frequency functions: A finite sample variance expression. Automatica, 42(4):589-600, May 2006.

[4] T. Kailath. Linear Systems. Prentice-Hall, Englewood Cliffs, New Jersey, 1980.

[5] J. Löfberg. Yalmip: A toolbox for modeling and optimization in MATLAB. In Proceedings of the CACSD Conference, Taipei, Taiwan, 2004.

[6] L. Ljung. System Identification: Theory for the User. Prentice-Hall, Englewood Cliffs, NJ, 2nd edition, 1999.

[7] L. Ljung and B. Wahlberg. Asymptotic properties of the least-squares method for estimating transfer functions and disturbance spectra. Advances in Applied Probability, 24(2):412-440, 1992.

[8] L. Ljung and Z.D. Yuan. Asymptotic properties of black-box identification of transfer functions. IEEE Trans. Automatic Control, 30(6):514-530, 1985.

[9] R.K. Mehra. Optimal input signals for parameter estimation in dynamic systems - Survey and new results. IEEE Transactions on Automatic Control, 19(6):753-768, 1974.

[10] C. R. Rojas, J. C. Agüero, J. S. Welsh, and G. C. Goodwin. On the equivalence of least costly and traditional experiment design for control. Automatica, 44:2706-2715, November 2008.

[11] C. R Rojas, J. S. Welsh, and J. C. Agüero. Fundamental limitations on the variance of parametric models. IEEE Transactions on Automatic Control, pages 1077-1081, 2009.

[12] P. Stoica, J. Li, and Y. Xie. On probing signal design for mimo radar. IEEE Transactions on Signal Processing, (8):4151-4161, 2007.

[13] P. Stoica and T. Marzetta. Parameter estimation problems with singular information matrices. IEEE Transactions on Signal Processing, pages 87-90, 2001.

[14] P. Stoica and R. Moses. Spectral Analysis of Signals. Prentice Hall Press, 2005.

[15] B. Wahlberg, M. Barenthin Syberg, and H. Hjalmarsson. Nonparametric methods for L2-gain estimation using iterative experiments. Automatica, 46(8):1376-1381, August 2010.

[16] B. Wahlberg, H. Hjalmarsson, and P. Stoica. On the performance of optimal input signals for frequency response estimation. IEEE Transactions on Automatic Control, July 2010. Submitted. 